\title{
11. A Case of Intracerebral Plasmacytoma
}

\author{
Hirobumi Ryu, Akira Saito, Yoshiharu Imazeki and Susumu Matsui \\ Dept. of Surgery, School of Medicine, Showa Univ.
}

Koji Tashiro, Masao Tei and Tetsu Kataoka

Dept. of Pathology, School of Medicine, Showa Univ.

A rare case of intracerebral plasmacytoma in a male aged 47 , that produced sudden unconsciousness for an hour with nuchal rigidity and radiating pain to the scapula was presented. Subsequently he developed his right side paresis for a while and 5 days later he had second attack of unconsciousness with mild speech disturbance.

Since these episodes he has had same attack three times. Finally he was suspected of brain tumor and transfered to us for further evaluation.

Bilateral carotid angiogram revealed only mild sclerosis, however pneumoencephalogram showed a over thumb size defect in the transitional area from posterior to inferior horn of the left lateral ventricle.

By vertebral angiogram a tumor like contour (about $3 \mathrm{~cm}$ in diameter) was visualized in the same area mentioned above. Partial exstirpation of the tumor was performed.

\author{
12. Metastatic Struma in the Frontal Lobe. \\ Report of a Cace \\ Jun-ichi Kawafuchi, Fumikazu Takeda and Norio Kohno \\ Department of Neurosurgery, School of Medicine, Gunma University \\ Susumu KaneKo \\ Ist Department of Surgery, School of Medicine, Gunma University
}

A brain tumor located in the frontal lobe was extirpated and histologically proved to be metastasis of the thyroid tumor. But the primary site of the tumor in the thyroid was not clinically detected. After eight months of follw up, a small carcinoma was found in the thyroid and resected.

The patient was 33-years-old housewife. She suffered from failing vision for three months prior to admission. Bilateral choked disk was noticed. The physical examination was negative. Carotid angiography revealed a tumor in the right frontal lobe. Craniotomy was performed, and a cystic tumor, containing $50 \mathrm{cc}$ of colloidal fluid, was extirpated. Histologically, colloid-producing follicular struc- 
tures with papillary pattern were noted along the wall of the cyst, and diagnosed papillary adenoma of thyroid origin. The colloidal fluid in the cyst contained $110 \gamma / \mathrm{dl}$ of PBI, of which $9.6 \%$ was organic. The tumor was biochemically an abnormal thyroid tissue. Nothing abnormal was noticed in her cervical region. $\mathbf{I}^{131}$-scintigram appeared normal, and no abnormal uptake of $\mathbf{I}^{131}$ was found in the head and chest. She discharged a month after operation and followed up. Eight months after craniotomy, repeated ${ }^{131}$-scintigram of the thyroid revealed a cold area in the left lobe. She again admitted and operation was performed. A small nodule, measuring $4 \mathrm{~mm}$ in diameter, was discovered in the left lobe of the thyroid with minute metastasis in an adjacent lymph node. Left radical lobectomy was performed. Histologically, the nodule in the thyroid was papillary adenocarcinoma, less differentiated than the metastatic frontal lobe tumor, and considered as the primary lesion.

\title{
13. A Postoperative Long Survival Case of Intracranial Sarcoma. Report of a Case
}

\author{
Naoki Kageyama and Kimiyuki IKedA \\ Dept. of Neurosurgery, Kansai Medical College \\ Hisashi OYA \\ Pediatric Dept., Kitano Hospital
}

Most of intracranial sasrcomas recur within 1-2 years after surgical removal and the prognosis is almost always unfavorable. A case of an intracranial sarcoma who has survived for 6 and a half years postoperatively is reported. The clinical history is as follows:

Case report: The patient was an 11-year-old girl who was admitted to the 1st Surgical Division of Kyoto University Hospital on March 14, 1961 because of frequent spells of awkwardness and weakness of the right half of the body accompanied by paresthetic feeling in the right lower extremity for about 5 month duration. Since four months ago she has complained nausea and headache.

Neurological examination revealed tenderness on percussion in the left parietal area, right spastic hemiparesis and positive bilateral Babinski sign. Skull plain films showed a calcified mass of walnut-size in the left parietal area. Left carotid angiogram revealed a space occupying lesion in the corresponding area of the calcification. Left parietal craniotomy was done on March 18, 1961. A sharply demarcated calcified tumor was found in the parietal parasagittal area and partly attached to the dura. The tumor was carefully separated from the surrounding brain tissue and extirpated in toto en block. After the operation the patient has 